\title{
Covariant model for dynamical quark confinement
}

\author{
Helmut Haberzettl \\ Center for Nuclear Studies, Department of Physics, \\ The George Washington University, Washington, D.C. 20052
}

(7 February 1994)

\begin{abstract}
Based on a recent manifestly covariant time-ordered approach to the relativistic many-body problem, the quark propagator is defined by a nonlinear Dyson-Schwinger-type integral equation, with a one-gluon loop. The resulting energy-dependent quark mass is such that the propagator is singularity-free for real energies, thus ensuring confinement. The self-energy integral converges without regularization, due to the chiral limit of the quark mass itself. Moreover, the integral determines the low-energy limit of the quark-gluon coupling constant, for which a value of $g^{2} / 4 \pi=4.712$ is found.
\end{abstract}

PACS number: 12.38.Aw

Typeset using REVTEX 
In the absence of a direct derivation of the dynamical mechanism of quark confinement from the QCD Lagrangian, a number of different model approaches have been considered in recent years to achieve confinement [1 80. (For a recent extensive review of related questions and further references, see [9].) Conceptually arguably one of the most elegant mechanisms to this end is based on the idea that the quark propagator should not have a pole, a mechanism which was proposed already twenty years ago [10,11] and which has been implemented in Refs. [1 8, 8,8]. In this letter, we want to present a model of quark confinement along similar lines, i.e., we will derive a quark propagator without any singularities for real energies.

Our model is based on the cluster-dynamical approach to the relativistic many-body problem proposed in Refs. [12, [13]. This scattering formulation is a time-ordered one which is manifestly covariant under an off-shell modification $\mathcal{M}$ of the Lorentz transformation $\mathcal{L}$. The transformation $\left(e^{\prime}, \mathbf{q}^{\prime}\right)=\mathcal{M}(e, \mathbf{q})$ of an arbitrary four-vector $(e, \mathbf{q})$ describing a cluster with three-momentum $\mathbf{q}$ and off-shell energy $e$ is defined by relating the three-momenta $\mathbf{q}^{\prime}$ and $\mathbf{q}$ in two different frames by Lorentz transformations involving only the respective on-shell energies $\omega^{\prime}$ and $\omega$, i.e., $\left(\omega^{\prime}, \mathbf{q}^{\prime}\right)=\mathcal{L}(\omega, \mathbf{q})$, where $\omega=\omega(m, \mathbf{q})=\left(m^{2}+\mathbf{q}^{2}\right)^{1 / 2}$, etc., with $m$ being the mass. The relation between off-shell energies $e^{\prime}$ and $e$ is then defined by

$$
e-\omega(m, \mathbf{q})=e^{\prime}-\omega\left(m, \mathbf{q}^{\prime}\right),
$$

similar to Galilei transformations in Euclidean space. The modified transformations $\mathcal{M}$, therefore, leave the differences between on- and off-shell energies invariant, and reduce to $\mathcal{L}$ when going on-shell. Performing then all internal integrations with the appropriate covariant integral measure [cf. discussion after Eq. (6) below], the time-ordered cluster formalism of Refs. [12,13] is manifestly covariant under $\mathcal{M}$ and hence Lorentz-covariant for arbitrary onshell matrix elements. We note that, while $\mathcal{M}$ appears as a nonlinear transformation of fourvectors $(e, \mathbf{q})$, it can be can be understood [13] as a covariant four-dimensional projection of a linear five-dimensional extension of the Lorentz transformation $\mathcal{L}$, i.e., Minkowski space is a hypersurface of an underlying five-dimensional manifold whose extra, fifth, dimension is interpreted as the off-shell energy variable with transformation properties defined in (11), while the usual Minkowski-space energy component is mapped onto the mass shell.

The simplest possible way of defining a time-ordered quark propagator in the approach of Refs. [12,13] is given by the one-gluon-loop expansion depicted in Fig. 1. The nonlinearitystructure of this integral equation is similar to a Dyson-Schwinger equation [14,9], albeit in a time-ordered framework. In the center-of-momentum system (CMS), the resulting dressed (time-ordered) quark propagator is given by

$$
\begin{aligned}
t(E-m+i 0) & \equiv \frac{\mathcal{P}(m, \mathbf{0})}{E-m+i 0} \\
& =\frac{\mathcal{P}(m, \mathbf{0})}{(E-M+i 0) Z-\Sigma(E)},
\end{aligned}
$$

where the first identity is a definition of the quark mass $m$; $M$ is the bare mass, $Z$ the renormalization constant and $\Sigma$ the self-energy given by the gluon-loop bubble of Fig. 1 . $\mathcal{P}(m, \mathbf{q})=(\not q+m) / 2 m$ is the covariant on-shell spin- $1 / 2$ projector, i.e., $q^{\mu}=(\omega, \mathbf{q})$. (We recall in this context that in the present formalism [12,13] all spin degrees of freedom are described with on-shell energies, as a consequence of the particular off-shell continuation 
described above.) Equation (2) defines the quark mass as a dynamical quantity. As an invariant, it must be an implicit function of itself, $m=m(E-m)$, where $E-m$ is an invariant according to (四), hence $t(E-m+i 0)$ is manifestly covariant.

In the CMS frame, the explicit energy dependence [15] of $m(E-m) \rightarrow m(E)$ is readily found from (2). Requiring in particular that the mass $m(E)$ be a positive semidefinite quantity for all energies and using the result (to be shown below) that $\Sigma(E) \geq 0$ for all energies, it follows that the renormalization constant $Z$ must be unity, $Z=1$, and hence

$$
m(E)-M=\Sigma(E)
$$

If we suppose for the moment that $m(E)$ is indeed such that the quark propagator (2) is free of singularities for real energies, i.e., that $E-m(E)$ is always negative, then we may introduce a new function $m_{0}(E)$ by writing $\Sigma(E)=-m_{0}^{2}(E) /(E-m)$; we then find

$$
m(E)-M=\frac{E-M}{2}+\sqrt{\left(\frac{E-M}{2}\right)^{2}+m_{0}^{2}(E)} .
$$

For large negative energies, $m(E)$ approaches the bare mass $M$ from above if we assume that $m_{0}^{2}(E) /|E-M|$ goes to zero, i.e., $M$ provides a lower bound for the dynamical mass $m(E)$. In the following, we will put the bare mass $M=0$, in other words, we require that the dynamical quark mass approach its chiral limit for large negative energies,

$$
m(E)=\frac{E}{2}+\sqrt{\left(\frac{E}{2}\right)^{2}+m_{0}^{2}(E)} .
$$

Our results will verify that $m(E)$ has indeed the energy behavior given by this equation.

To this end, we take the quark-gluon vertex to be undressed and given simply by $g \gamma^{\mu} \lambda_{a} / 2$, where $g$ is the coupling constant and $\lambda_{a}$ and $\gamma^{\mu}$ are the usual color and Dirac matrices, respectively. From (3), with $M=0$, it then follows, using the rules of Refs. 12, 13 for evaluating the self-energy bubble $\Sigma(E)$ and keeping in mind the invariance property (1), that

$$
m(E)=i \frac{4}{3} \frac{g^{2}}{(2 \pi)^{4}} \int_{-\infty}^{+\infty} d e \int d^{3} q \frac{m(E-e-\omega+m)}{2 q \omega(m, \mathbf{q})} \frac{N}{(E-e-\omega+i 0)(e-q+i 0)},
$$

where the variables of Fig. 1 were used. Recall here that in the present approach 12, 13 three-momenta are calculated with on-shell energy parameters in the Lorentz transformations, and $d^{3} q \mathrm{~m} / 2 q \omega$ is the corresponding covariant integral measure, with normalization factors $2|\mathbf{q}| \equiv 2 q$ and $\omega / m$ due to the gluon and the quark, respectively. Note that all masses here are expressed in the CMS notation as explicit functions of the corresponding off-shell energy; hence, in the integrand the quark mass and the associated on-shell 117 energy are coupled functions of each other and implicit functions of themselves, i.e., $m=m(E-e-\omega+m)$ and $\omega=\omega(m(E-e-\omega+m), \mathbf{q})$. The remaining ratio arises from the energy convolution of the time-ordered quark and gluon propagators whose detailed variable dependence follows from the invariance requirement (1). $N$ is the spinor matrix element,

$$
N=\bar{u}(\mathbf{0}, s) \gamma_{\mu} \frac{\varnothing+m}{2 m} N^{\mu \nu} \gamma_{\nu} u(\mathbf{0}, s)
$$


where $Q^{\mu}=(\omega,-\mathbf{q})$ is the on-shell four-momentum of the dressed quark in the loop of Fig. 1 and $\bar{u}, u$ are Dirac spinors of the quark external to the self-energy loop with third spin component s. $N^{\mu \nu}$ is given by the numerator of the time-ordered gluon propagator,

$$
G^{\mu \nu}(e-q+i 0)=\frac{N^{\mu \nu}}{e-q+i 0}
$$

We treat the gluon here as an undressed particle similar to a bare photon. The question then arises how to incorporate gluon confinement. This is, of course, a very complicated and as yet unsolved problem (for recent reviews, see [9, 16], and references therein). We deal with it here by what we consider the simplest possible confinement mechanism known already from QED: discarding transverse contributions, we restrict the gluons to longitudinal and scalar (or, temporal) ones, which are unobservable the same way longitudinal and scalar photons are unobservable directly. We can offer no deeper justification of this procedure other than that it works for the present purpose. Hence, in Feynman gauge one has

$$
N^{\mu \nu}=\beta^{\mu} \beta^{\nu}-\eta^{\mu} \eta^{\nu}
$$

where $\eta^{\mu}=(1,0)$ and $\beta^{\mu}=(0, \mathbf{n})$, with $\mathbf{n}$ being the unit vector defined by the gluon three-momentum $\mathbf{q}=q \mathbf{n}$. One finds then that simply $N=-1$.

In evaluating (6), we assume now that there are no singularities of the integrand in the lower half of the complex $e$ plane other than the one at $e=q-i 0$ (cf. discussion below). The residue of the energy integration then yields

$$
m(E)=-\frac{g^{2}}{3 \pi^{2}} \int_{0}^{\infty} d q q \frac{m(E-q-\omega+m)}{\omega(m(E-q-\omega+m), \mathbf{q})} \frac{1}{E-q-\omega(m(E-q-\omega+m), \mathbf{q})}
$$

where we have also carried out the trivial angle integrations and dropped the $i 0$ in the denominator of the quark propagator in anticipation of the fact that the mass $m(E)$ will indeed turn out to be confining. This equation is the main result of the present letter; it provides a highly nonlinear self-consistency equation for the dynamical quark mass $m(E)$. Note that, in view of Eq. (5), for large momenta $q$ the integrand behaves like $m_{0}^{2}(-2 q) / q^{2}$, in other words, the integral exists. [The numerical results discussed below verify that $m_{0}^{2}(-2 q)$ goes to a constant for $q \rightarrow \infty$.] The convergence of the integral thus is achieved by the chiral limit itself, without any cutoff whatsoever.

Note also that Eq. ([10) does not fix any energy scale: using an arbitrary scale $\Lambda$, the corresponding solution $m_{\Lambda}(E)$ may be rewritten as $m_{\Lambda}(E)=\Lambda \mu_{1}(E / \Lambda)$, where the functional form of the dimensionless $\mu_{1}$ is the same as $m_{\Lambda}$. Furthermore, $\mu_{1}$ can be rescaled with an arbitrary (positive, dimensionless) constant $\lambda$ according to $\mu_{1}(\epsilon)=\lambda \mu_{1 / \lambda}(\epsilon / \lambda)$. This is just the scaling behavior of the renormalization group. The energy scale must be fixed by other considerations, e.g., by the value of $m(E)$ at $E=0$, where $m(0)=m_{0}(0)$, which should be roughly given by the phenomenological constituent mass of the quarks, around $300 \mathrm{MeV}$, which in turn is about equal to $\Lambda_{Q C D}$.

A trivial solution of Eq. (10) is the chiral limit itself, $m(E)=0$. Since the right-hand side of Eq. (10) is a simple one-dimensional integral, other solutions can be obtained by an iterative numerical procedure. In view of the high nonlinearity, however, this is not as easy as it may look if one would like to find the complete set of solutions [18]. At present, 
therefore, we have made no attempt to do so but simply solved Eq. (10) by straightforward iteration. As a starting point, we chose $m(E)$ to be of the form (5) with $m_{0}$ as a constant, $m_{0}=1$, which fixes the energy scale. Both converged solution and starting function are given in Fig. 2. As can be seen, the solution always stays above the line given by the pole condition $m=E$. This solution thus is indeed confining, approaching the line $m=E$ only asymptotically, as it must if quarks are to be free asymptotically. Note also that the starting function is an excellent approximation of $m(E)$ for negative energies and around $E=0$. Since Eq. (10) is a nonlinear eigenvalue condition, the solution of Fig. 2 is found to correspond to a fixed coupling-constant eigenvalue of

$$
\frac{g^{2}}{4 \pi}=4.712
$$

a value numerically accurate to better than $0.1 \%$. With a straightforward iteration of Eq. (10), no other eigenvalue could be found. However, experience with iterative solutions of other nonlinear equations [18] does not rule out the existence of other eigenvalues.

As the low-energy limit of the QCD coupling constant, the value in (11) is a bit on the low side. We emphasize, however, that it was obtained without taking into account that the QCD coupling constant should be a function of the gluon momentum $q$ in (10). Solving Eq. (10) with a decreasing running coupling constant $\alpha_{s}\left(q^{2}\right)$ with a reasonable range of parameters, one finds, first, that $\alpha_{s}(0)=g^{2} / 4 \pi$ may increase by up to about an order of magnitude — which does cover the range of expected low-energy values — and, second, that the corresponding solutions $m(E)$ approach the asymptotic-freedom limit of $m(E)=E$ faster than for the case shown in Fig. 1. In view of the relative simplicity of the present model, we do not want to dwell on any details of this investigation concerning a running coupling constant; nevertheless, we find it encouraging that the trends go in the right direction.

Our result verifies that the dynamical quark mass can indeed be written in the form of Eq. (5). Since everything is completely known in the defining equation (10), in principle it should be possible to find the analytic properties of $m(z)$ for an arbitrary complex energy $z$. In practice, this is complicated very much by the high degree of nonlinearity. Equation (5) suggests that $m(z)$ has square-root cuts in the complex plane with branch points given by the implicit condition $z=2 m(z)$. Various approximations and numerical studies seem to indicate that this condition cannot be met in the integrand of Eq. (6) for complex values of $e$ in the lower half of the complex plane, thus justifying the derivation of Eq. (10). We mention, however, that we do not consider our findings to be entirely conclusive in this respect.

In summary, we have presented here the simplest-possible one-gluon-loop expansion for the quark propagator in the covariant time-ordered formulation of the relativistic manybody problem of Refs. [12, 13]. We find a confining solution where the chiral limit of the dynamical quark mass $m(E)$ itself provides the necessary cutoff to make the self-energy integral convergent.

The author would like to thank Professors D. Schütte and H. Petry for interesting discussions. This work was supported in part by the U.S. Department of Energy, Grant No. DE-FG05-86-ER40270. 


\section{REFERENCES}

[1] M. Stingl, Phys. Rev. D 34, 3863 (1986); ibid. D 36, 651 (1987).

[2] C. M. Shakin, Ann. Phys. (NY) 192, 254 (1989).

[3] V. Sh. Gogokhia, Phys. Lett. B224, 177 (1989).

[4] R. Oehme, Phys. Lett. B232, 498 (1989).

[5] P. C. Tandy and M. R. Frank, Aust. J. Phys. 44, 181 (1991); Phys. Rev. C 43, 2808 (1991).

[6] F. Gross and J. Milana, Phys. Rev. D 43, 2401 (1991); ibid. D 45, 969 (1992).

[7] C. J. Burden, C. D. Roberts, and A. G. Williams, Phys. Lett. B285, 347 (1992).

[8] M. Buballa and S. Krewald, Phys. Lett. B294, 19 (1992); M. Buballa, Ph.D. Thesis (Universität Bonn, June 1993), KFA Jülich Report Jül-2788 (1993).

[9] C. D. Roberts and A. G. Williams, in "Progress in Particle and Nuclear Physics" (Pergamon Press, Oxford, 1994) [in press].

[10] G. Preparata, Nucl. Phys. B80, 299 (1974).

[11] R. Fukada and T. Kugo, Nucl. Phys. B117, 250 (1976).

[12] H. Haberzettl, Phys. Rev. C 47, 1237 (1993).

[13] H. Haberzettl, Phys. Rev. C 49 (1994) [in press].

[14] F. J. Dyson, Phys. Rev. 75, 1736 (1949); J. Schwinger, Proc. Natl. Acad. Sci. (USA) 37, 452 and 455 (1951).

[15] For simplicity, we do not use different function names for the mass depending implicitly on $E-m$ or explicitly on $E$ since it will be obvious from the context what is meant.

[16] C. D. Roberts, in "QCD Vacuum Structure", eds. H. M. Fried and B. Müller (World Scientific, Singapore, 1993).

[17] Obviously, if the notion that quarks are confined because their propagator does not have a pole is to make sense, the word "on-shell" has to be taken with a grain of salt. Thus the "on-shell" energy for a quark depends on its off-shell energy through the off-shell dependence of its dynamical mass.

[18] As a rather trivial but still quite illustrative example, we recall that a quadratic equation $a x^{2}+b x+c=0$ can be written as $x=-c /(b+a x)$ and solved by iteration. While, in general, the iteration will converge very rapidly, it will provide only one of the two solutions of the quadratic equation, namely the one smaller in magnitude. To get the larger one, one must iterate $1 / x \equiv y=-a /(b+c y)$. 


\section{FIGURES}

FIG. 1. Nonlinear one-gluon-loop integral equation of the time-ordered dressed quark propagator (solid line with dot) in terms of bare quark propagator (solid line) and gluon loop (cork-screw line); time proceeds from right to left. The off-shell energies and three-momenta are given in the center-of-momentum system of the dressed quark; $e$ and $\mathbf{q}$ are the loop-integration variables of Eq. (6).

FIG. 2. Iterative solution of Eq. (10) (solid line). Both energy $E \rightarrow \epsilon$ and mass $m \rightarrow \mu$ are mapped here by the same transformation $x \rightarrow 2 \arctan (x / s) / \pi$ from $(-\infty,+\infty)$ to the interval $(-1,+1)$; in the graph, the parameter $s$ is arbitrarily chosen as $s=5$. The energy scale is fixed by requiring $m(0)=1$ [i.e., $\mu(0)=0.1257$ after transformation]. The long-dashed line is given by Eq. (5) with $m_{0}(E)$ fixed at $m_{0}(E)=1$. The short-dashed straight diagonal line corresponds to the pole condition $m=E$. 